\title{
Observation of electron driven quasi-coherent modes and their connection with core intrinsic rotation in KSTAR ECH and ohmic L-mode plasmas
}

J. A. Lee (이지훈 ), W. Lee, J. M. Kwon, S. H. Ko, J. Leem, G. S. Yun, H. K. Park, Y. S. Park, K. W. Kim, N. C. Luhmann, and KSTAR Team

Citation: Physics of Plasmas 25, 022513 (2018);

View online: https://doi.org/10.1063/1.5008468

View Table of Contents: http://aip.scitation.org/toc/php/25/2

Published by the American Institute of Physics

\section{Articles you may be interested in}

Damping of zonal modes through turbulent momentum transport

Physics of Plasmas 25, 022505 (2018); 10.1063/1.5018511

Investigation of instabilities and rotation alteration in high beta KSTAR plasmas

Physics of Plasmas 24, 012512 (2017); 10.1063/1.4974170

Predicting rotation for ITER via studies of intrinsic torque and momentum transport in DIII-D

Physics of Plasmas 24, 056113 (2017); 10.1063/1.4979194

Investigation of intrinsic toroidal rotation scaling in KSTAR

Physics of Plasmas 24, 072510 (2017); 10.1063/1.4991397

Symmetry breaking induced by the parity change in global electromagnetic ion temperature gradient modes

Physics of Plasmas 25, 012505 (2018); 10.1063/1.5006981

Response of a core coherent density oscillation on electron cyclotron resonance heating in Heliotron $\mathrm{J}$ plasma Physics of Plasmas 25, 012513 (2018); 10.1063/1.5007903

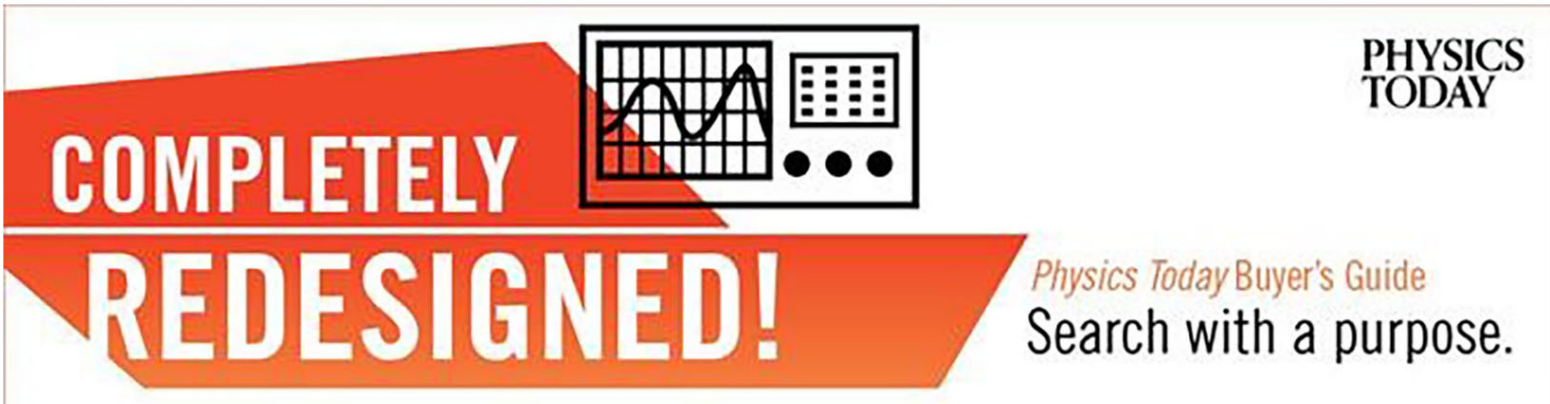




\title{
Observation of electron driven quasi-coherent modes and their connection with core intrinsic rotation in KSTAR ECH and ohmic L-mode plasmas
}

\author{
J. A. Lee (이지훈), ${ }^{1}$ W. Lee, ${ }^{2, a)}$ J. M. Kwon, ${ }^{2}$ S. H. Ko, ${ }^{2}$ J. Leem, ${ }^{1}$ G. S. Yun, ${ }^{1}$ H. K. Park, ${ }^{3}$ \\ Y. S. Park, ${ }^{4}$ K. W. Kim, ${ }^{5}$ N. C. Luhmann, Jr., ${ }^{6}$ and KSTAR Team \\ ${ }^{1}$ Department of Physics, Pohang University of Science and Technology, Pohang, Gyeongbuk 37673 , \\ South Korea \\ ${ }^{2}$ National Fusion Research Institute, Daejeon 34133, South Korea \\ ${ }^{3}$ Department of Physics, Ulsan National Institute of Science and Technology, Ulsan 44919, South Korea \\ ${ }^{4}$ Department of Applied Physics and Applied Mathematics, Columbia University, New York, New York 10027, USA \\ ${ }^{5}$ School of Electrical and Computer Engineering, Kyungpook National University, Daegu 41566, South Korea \\ ${ }^{6}$ Department of Electrical and Computer Engineering, University of California at Davis, Davis, \\ California 95616, USA
}

(Received 6 October 2017; accepted 30 January 2018; published online 15 February 2018)

Quasi-coherent (QC) modes, known as a type of the trapped electron mode (TEM) turbulence, have been measured in the outboard core region of low-density electron cyclotron resonant heating $(\mathrm{ECH})$ injected and ohmically heated L-mode plasmas on the Korea Superconducting Tokamak Advanced Research (KSTAR) device. It appears that QC modes of $20-60 \mathrm{kHz}$ occur or strengthen with an increase of the electron temperature to ion temperature ratio, but weaken or are fully suppressed by increased density/collisionality. Toroidal rotation shear, which is strongly related to the density/collisionality, also seems to stabilize the QC modes. Linear gyrokinetic simulations indicate that TEM is the most unstable mode at low densities where the QC modes are observed for both the ECH and ohmic plasmas. At high densities where the QC modes are suppressed, the most unstable mode is the ion temperature gradient (ITG) mode for the ECH plasmas but still TEM for the ohmic plasmas. In the ECH plasmas, it is found that the direction of the core toroidal intrinsic rotation is gradually reversed from the co-current to counter-current direction and the QC mode is suppressed as the line-averaged density increases, which can be explained by the transition of dominant micro-turbulence (TEM to ITG). However, in the ohmically heated plasmas, the acceleration of the core toroidal rotation is not fully explained by the TEM-ITG transition since the direction of the core toroidal rotation is counter-current direction when the QC mode is observed. Furthermore, the core toroidal rotation is accelerated to the counter-current direction even the line-averaged density decreases. Published by AIP Publishing. https://doi.org/10.1063/1.5008468

\section{INTRODUCTION}

To achieve fusion ignition, it is necessary to suppress microscale turbulences which degrade plasma confinement. ${ }^{1,2}$ There are several types of micro-turbulence: ion temperature gradient (ITG) modes, ${ }^{3-5}$ trapped electron modes (TEM), ${ }^{6,7}$ electron temperature gradient (ETG) modes, ${ }^{8,9}$ etc. Theoretical works have predicted that the scale of ETG is distinct from those of ITG/TEM because the scale of ETG is around $k_{\perp} \rho_{i} \sim 10$, but the scale of ITG/TEM is around $0<k_{\perp} \rho_{i}<1,{ }^{10}$ where $k_{\perp}$ is the perpendicular wavenumber of turbulence and $\rho_{i}$ is the ion gyro-radius. But it is difficult to directly distinct ITG and TEM because the scales of ITG and TEM are similar. However, it is important to discriminating them because it is known that they produce different effects on the toroidal intrinsic rotation, ${ }^{11,12}$ impurity transport, ${ }^{13}$ particle transport, ${ }^{14}$ etc. For the study of ITG/ TEM, measuring quasi-coherent (QC) modes can be helpful because it is known that they appear in TEM dominant and low normalized collisionality regimes but suppressed in ITG dominant and high collisionality regimes. ${ }^{15-19}$ QC modes have been observed in several tokamak machines

\footnotetext{
${ }^{\text {a) }}$ Author to whom correspondence should be addressed: wclee@nfri.re.kr
}

such as T-10, ${ }^{15}$ JET,,${ }^{17}$ TEXTOR, ${ }^{17}$ Tore Supra,,${ }^{16-18}$ HL$2 \mathrm{~A}$, and $\mathrm{J}-\mathrm{TEXT}^{19}$ and have revealed characteristics of both coherent modes and broadband fluctuations. In power spectra of electron density fluctuations, the mode shows a wide bandwidth (tens of $\mathrm{kHz}$ ) around a peak frequency ranging from $30 \mathrm{kHz}$ to $150 \mathrm{kHz} .{ }^{16,19}$ The modes are ballooned at the low-field side near the midplane and have been observed at many different radial locations from the plasma core to the edge. Gyrokinetic simulations have shown that the most unstable modes are electron modes such as TEM for low-density regimes where QC modes were observed.

In this paper, we focus on the properties of QC modes measured by the microwave imaging reflectometer (MIR) system $^{20-22}$ and the connection between QC modes and intrinsic rotations in ohmically heated and electron cyclotron resonant heating $(\mathrm{ECH})$ plasmas. In order to explain the mechanism of intrinsic rotation, a theoretical model, which is based on TEM-ITG transition, was proposed. ${ }^{23,24}$ This model explains that the direction of the core toroidal rotation is cocurrent direction (clockwise when viewed from above in typical KSTAR plasmas) in TEM dominant regimes, but it changes to counter-current direction in ITG dominant regimes. 
This paper is organized as follows: The description of the MIR system is given in Sec. II, and the experiment set-up and plasma parameters are presented in Sec. III. Section IV shows the properties of QC modes and dominant modes predicted from the gyrokinetic simulation. The changes in the intrinsic core toroidal rotation direction by the increase of electron density are discussed in Sec. V and summary is presented in Sec. VI.

\section{THE MICROWAVE IMAGING REFLECTOMETER (MIR) ON KSTAR}

The MIR was developed to measure electron density fluctuations in $2 \mathrm{D}(4 \times 16$ in the radial and poloidal directions from 2014) near the midplane. The 2D measurement can be achieved by utilizing four-frequency probe beams and the array of vertically aligned 16 detectors. ${ }^{21}$ The fourfrequency X-mode probe beams reflect from four different cutoff layers in the plasma, whose radial locations depend on the local electron density and magnetic field strength. The space between the cutoff layers is determined by the probing frequency step of $1.8 \mathrm{GHz}$ and local electron density profile, and it is typically $1-5 \mathrm{~cm}$. The cutoff layer locations can be positioned from the plasma core to the edge in between discharges by controlling the probing frequencies from $78 \mathrm{GHz}$ to $96 \mathrm{GHz}$ or magnetic field strength or electron density. However, variations of the cutoff layer locations due to changes in the equilibrium electron density in a single discharge are often unintended and unavoidable.

Through the carefully designed optical system, each channel of the vertically aligned 16 detectors can receive the reflected beams from poloidally localized areas of the cutoff layers. The poloidal spot size on the cutoff layer, which is related to the upper limit of detectable wavenumbers, is $\sim 0.9 \mathrm{~cm}$ in FWHM, providing the maximum wavenumber of $\sim 4 \mathrm{~cm}^{-1}$. The space between adjacent poloidal channels is $\sim 0.6 \mathrm{~cm}$ so that the 16 channels cover $\sim 9 \mathrm{~cm}$ over the cutoff layers. The poloidal spot size and space are reasonably small for resolving ion gyro-scale fluctuations such as the ITG mode and TEM. The time resolution is typically $1 \mu \mathrm{s}$ (with $10 \mathrm{~s}$ acquisition) and can be reduced down to $0.25 \mu \mathrm{s}$ (with $2.5 \mathrm{~s}$ acquisition).

\section{EXPERIMENTAL SETUP}

For the study of QC mode properties, we analyze two different types of discharges: ECH injected L-mode discharges and ohmically heated L-mode discharges. In order to study the dependence of QC mode on the electron density and collisionality, we increased the line-averaged electron density using deuterium $\left(D_{2}\right)$ gas puffing. However, when we interpret the evolution of the QC mode and estimate the radial location of the mode in a single discharge in which the electron density changes, it has to be taken into account that the increased electron density can shift the radial locations of the MIR cutoff layers outwards as discussed in Sec. II. For instance, disappearance of a QC mode by gas puffing seen in the MIR spectrogram can be interpreted in two ways: (1) the QC mode is suppressed by the increased density/collisionality or (2) the cutoff layers move away from the region where the QC mode is unstable. Thus, similar discharges were reproduced and measurements with various probing frequencies were carried out to measure the density fluctuations in a wide region.

The plasma parameters were as follows: all of the discharges were limiter plasmas; the plasma shapes were a Dshape for the ECH discharges as shown in Fig. 1(a) and close to a circular-shape for the ohmic discharges as shown in Fig. 1 (b); the central toroidal magnetic fields were $2.5 \mathrm{~T}$ for all discharges; the plasma currents were $500 \mathrm{kA}$ for the ECH discharges and $800 \mathrm{kA}$ for the ohmic discharges; and the line-averaged electron densities were increased from $\sim 1.3$ to $\sim 2.6 \times 10^{19} \mathrm{~m}^{-3}$ for the ECH discharges and from $\sim 2.0$ to $\sim 3.6 \times 10^{19} \mathrm{~m}^{-3}$ for the ohmic discharges. $140 \mathrm{GHz}$ ECH was injected at $R \sim 179 \mathrm{~cm}$ and $Z \sim+25 \mathrm{~cm}$ for the discharge $\# 16481$ and $Z \sim+40 \mathrm{~cm}$ for the discharge \#16485. Figure 2 shows the time traces of the plasma parameters for the ECH discharges (\#16481 and \#16485) and ohmic discharges (\#16124 \#16126). The electron temperature $\left(T_{e}\right)$ profiles were measured by the electron cyclotron emission (ECE) radiome$\operatorname{ter}^{25}$ or Thomson scattering system. ${ }^{26}$ Note that the time histories of $T_{e}$ plotted at the second panels of Figs. 2(a) and 2(b) were measured by the ECE radiometer. The line-averaged electron densities $\left(\bar{n}_{e}\right)$ were measured by the millimeter-wave interferometer ${ }^{27}$ and far-infrared (FIR) interferometer, ${ }^{28}$ the electron density $\left(n_{e}\right)$ profiles by the Thomson scattering system; and the ion temperature $\left(T_{i}\right)$ and toroidal rotation velocity $\left(V_{t}\right)$ profiles by the charge exchange recombination spectroscopy (CES). ${ }^{29}$ For the CES measurement, short neutral beam injection (NBI) called as the beam blip was used with the pulse length of $20 \mathrm{~ms}$ for the ECH discharges and $10 \mathrm{~ms}$ for the ohmic discharges, and the repetition rate of $2 \mathrm{~Hz}$. Note that the supersonic molecular beam injection (SMBI) was also applied to the ECH discharges for the electron density control, but its effect was small compared to gas puffing.

\section{PROPERTIES OF THE QC MODES IN ECH AND OHMIC L-MODE DISCHARGES}

Recent studies ${ }^{16-19}$ have investigated the QC mode properties such as the suppression by the increased density/
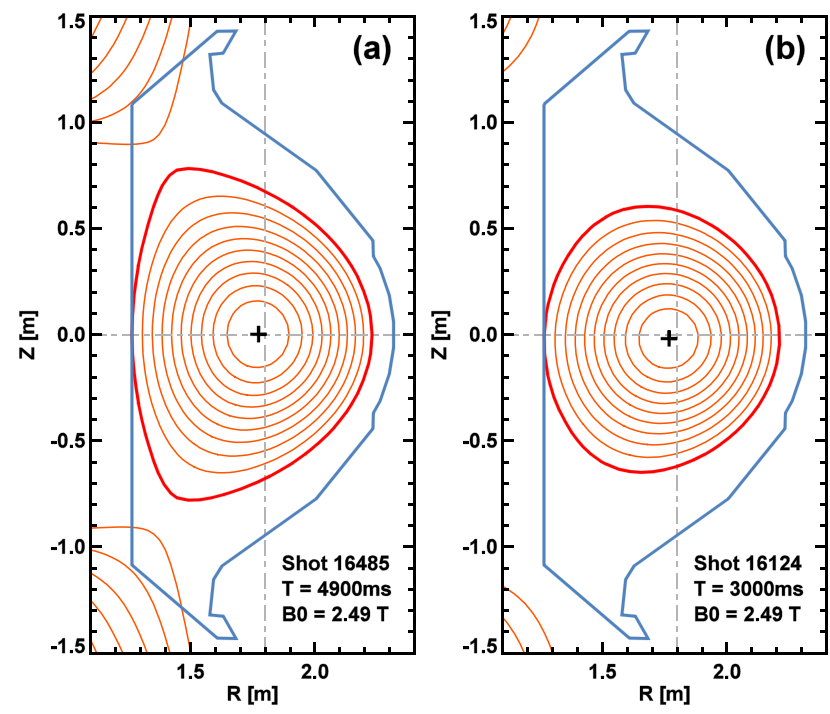

FIG. 1. Equilibrium magnetic flux surfaces reconstructed from EFIT calculations for an ECH discharge \#16485 (a) and an ohmic discharge \#16124 (b). 
(a) Shot 16481, 16485

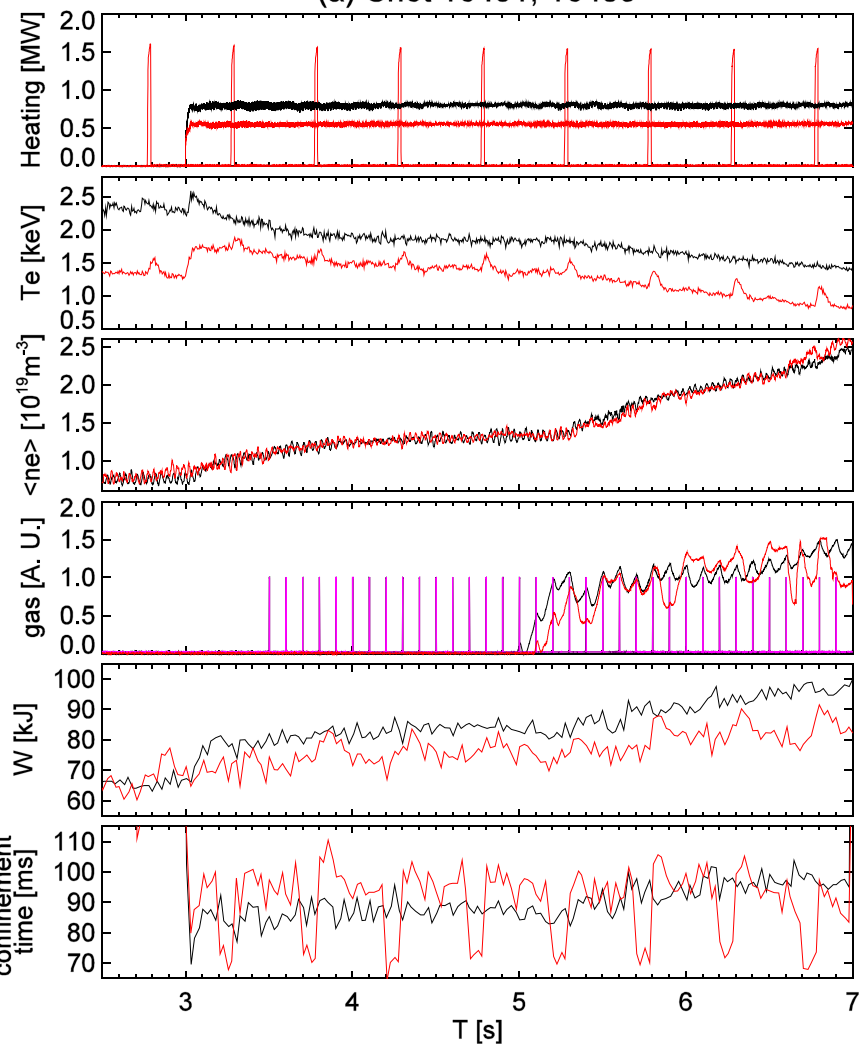

(b) Shot 16124, 16125, 16126
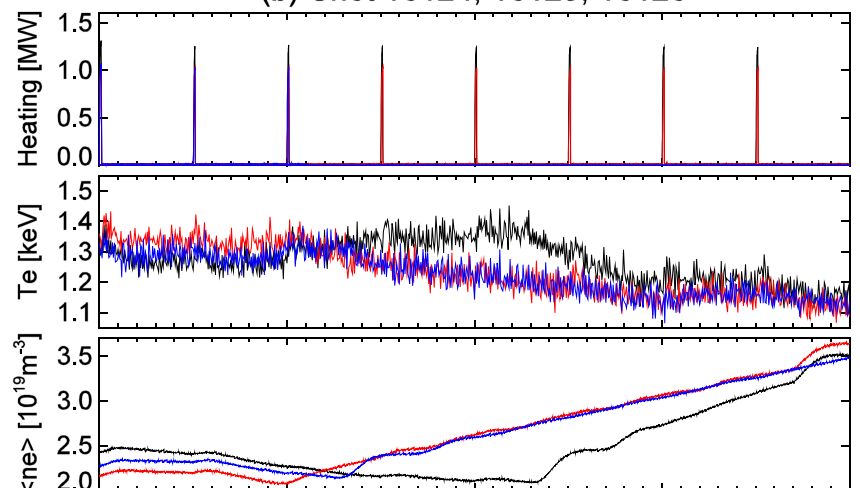

马ं 1.5

¿ 1.0

离 0.5

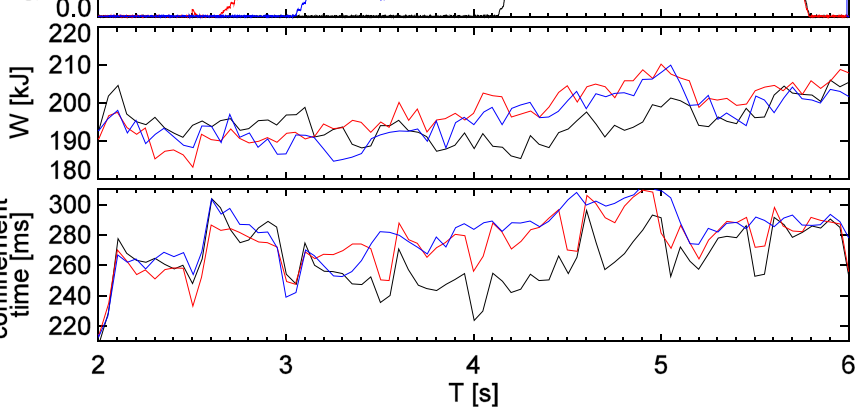

FIG. 2. Time histories of NBI blip and ECH powers, electron temperature at $\mathrm{R}=1.88 \mathrm{~m}$ (measured by the ECE radiometer), line-averaged electron density, deuterium gas puffing (and SMBI indicated by magenta color), stored energy, and energy confinement time for (a) the ECH discharges (\#16481: black and \#16485: red) and (b) ohmic discharges (\#16124: black, \#16125: red, and \#16126: blue).

collisionality, radial and poloidal localization, propagation in the electron diamagnetic direction in the plasma frame, and connection with TEM dominance in the region where the QC modes were measured obtained from gyro-kinetic simulations. All of these properties support that the QC mode is closely related to the TEM turbulence. In this paper, we study some of these properties for KSTAR ECH and ohmic L-mode plasmas and extend the property study by including the onset parameters for the QC mode in ECH plasmas, suppression by the toroidal rotation shear, and connection with the intrinsic toroidal rotation direction. We also investigate how the unstable mode changes in the plasmas in which QC mode evolved by increased density through linear gyrokinetic simulation using the GYRO code. ${ }^{30}$

\section{A. Overview of the QC modes}

In Fig. 3, the spectrogram of $n_{e}$ fluctuations measured by MIR shows that a QC mode appears when the ECH is switched on at $t=3.0 \mathrm{~s}$ and disappears as the line-averaged $n_{e}$ increases by gas puffing from $t=5.0 \mathrm{~s}$. Figure 4 shows the spectra of $n_{e}$ fluctuations at several radii for the ECH and ohmic discharges. The spectra have been taken from a similar line-averaged density, i.e., $1.3 \times 10^{19} \mathrm{~m}^{-3}$ at $t=5.2 \mathrm{~s}$ for the ECH discharges and $2.2-2.4 \times 10^{19} \mathrm{~m}^{-3}$ at $t=2.4 \mathrm{~s}$ for the ohmic discharges. The QC modes are radially localized in $184 \mathrm{~cm} \leq R \leq 191 \mathrm{~cm}$ or $0.18 \leq r / a \leq 0.33$ for the $\mathrm{ECH}$ discharges and $195 \mathrm{~cm} \leq R \leq 202 \mathrm{~cm}$ or $0.44 \leq r / a \leq 0.58$ for the ohmic discharges.

\section{B. Parameters destabilizing and stabilizing the QC modes}

Previous studies ${ }^{31-34}$ demonstrated that TEM can be excited by the increase of the $T_{e}$ to $T_{i}$ ratio $\left(T_{e} / T_{i}\right)$ and $T_{e}$ or $n_{e}$ gradient, while it can be suppressed by the collisionality, flow shear, magnetic shear, etc. And QC modes have been observed in the TEM dominant plasmas. ${ }^{16-19}$ Thus, it is logical to investigate the plasma parameters destabilizing or stabilizing the TEM turbulence as for the parameters for the QC mode.

The ECH discharges in Fig. 2(a) can be a good example for the study of the onset parameters for QC mode since a QC mode occurred immediately after the ECH injection

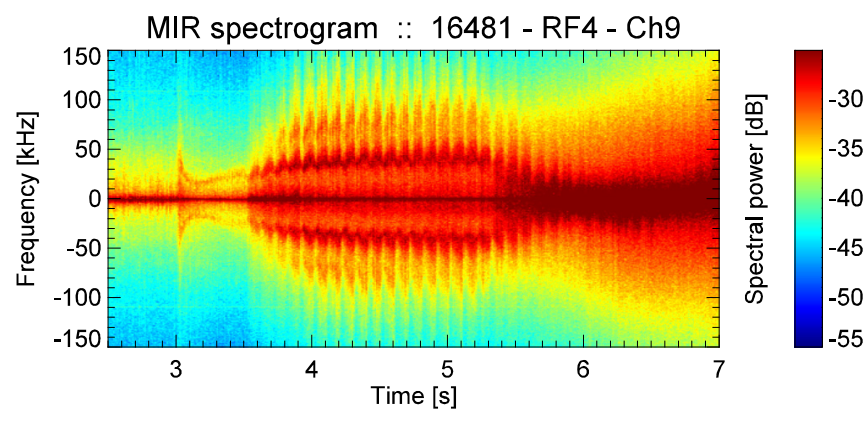

FIG. 3. Evolution of a QC mode in the ECH discharge \#16481 with the increase of the electron density. ECH was injected from $3.0 \mathrm{~s}$ to $7.0 \mathrm{~s}$. SMBI started from $3.5 \mathrm{~s}$ and $D_{2}$ gas puffing from $5.0 \mathrm{~s}$ for the increase of the electron density. 
(a)

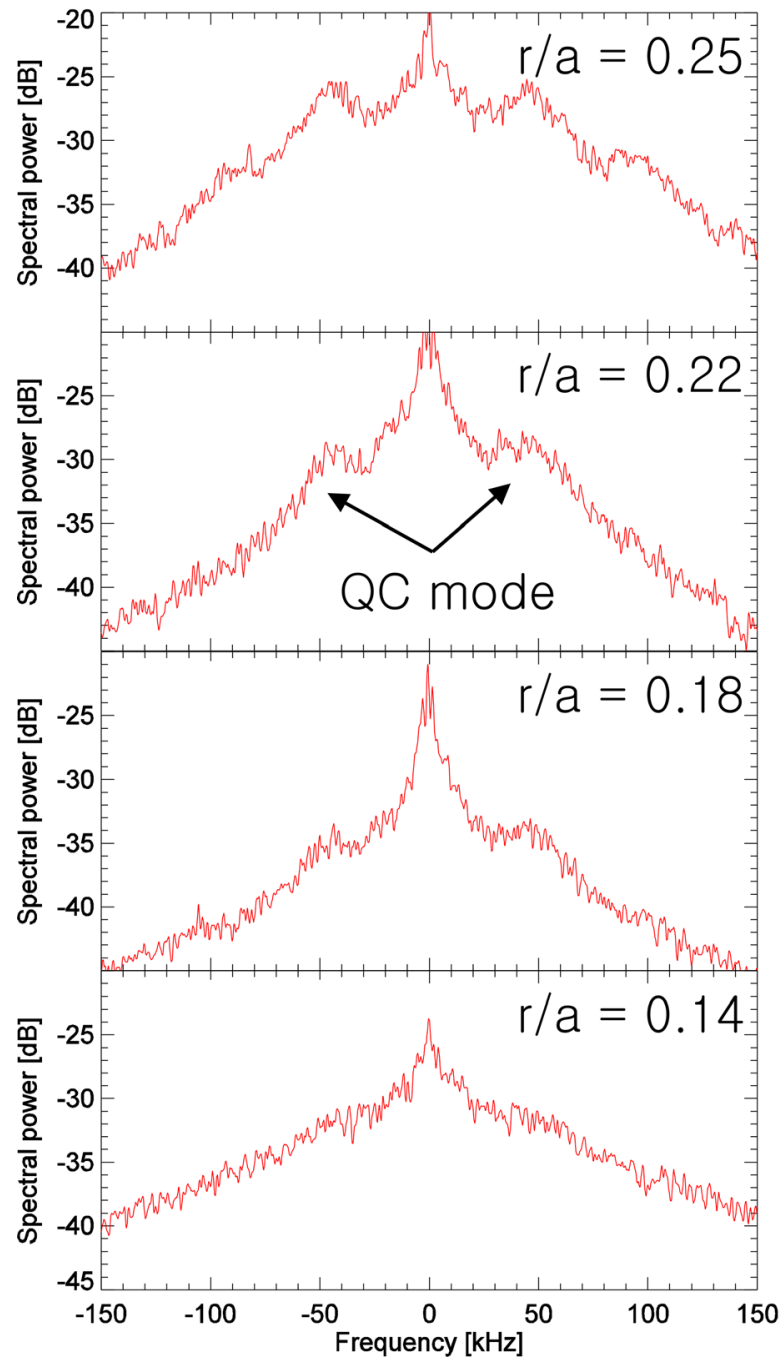

(b)

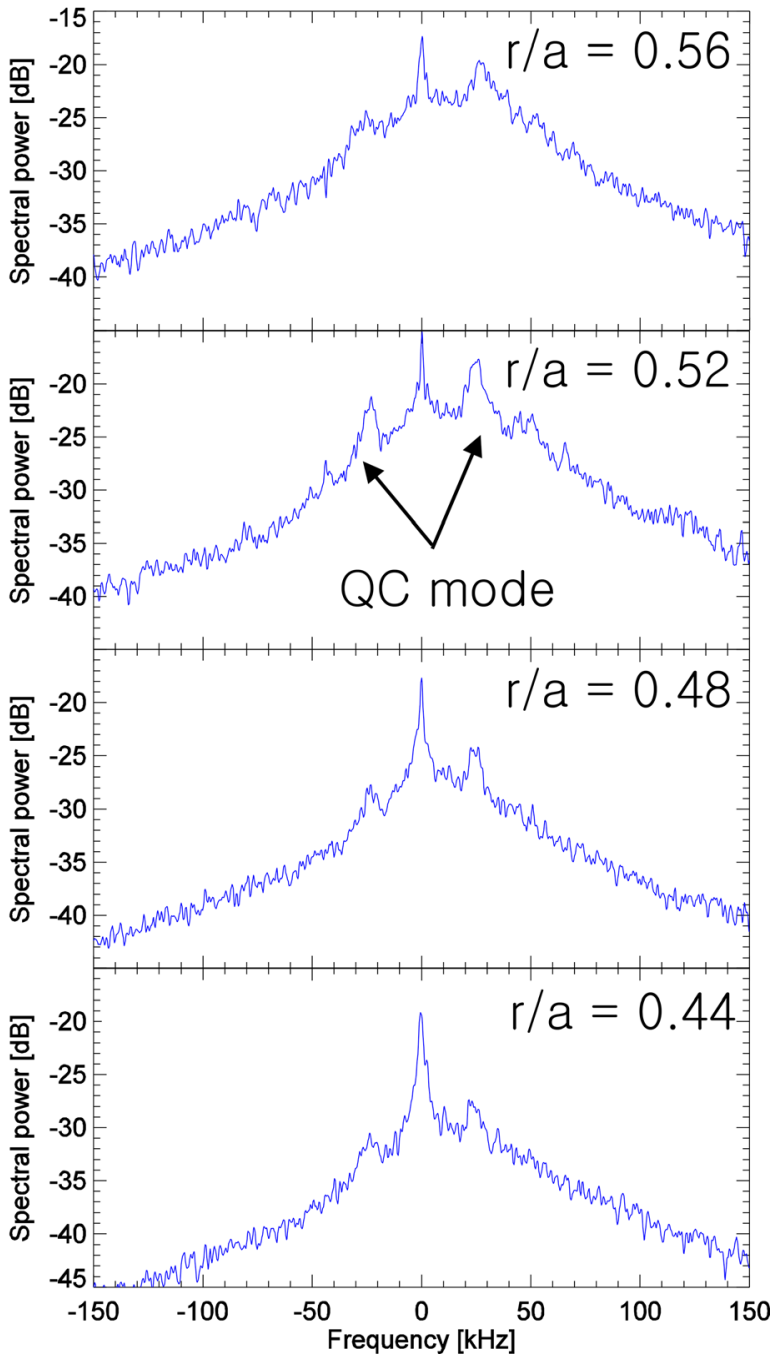

FIG. 4. Spectra of the density fluctuations at several locations for the ECH discharge \#16485 $\left(\mathrm{Ip}=500 \mathrm{kA}\right.$ and $\left.\bar{n}_{e}=1.3 \times 10^{19} \mathrm{~m}^{-3}\right)$ and the ohmic discharges $\# 16124$ and $16126\left(\mathrm{Ip}=800 \mathrm{kA}\right.$ and $\left.\bar{n}_{e}=2.2-2.4 \times 10^{19} \mathrm{~m}^{-3}\right)$.

(from $t=3.0 \mathrm{~s}$ ) as shown in Fig. 3. Figures 5(a)-5(d) show the profiles of $n_{e}, T_{e}, T_{i}$, and $T_{e} / T_{i}$ for an ECH discharges $\# 16481$ before $(t=2.8 \mathrm{~s})$ and after $(t=3.3 \mathrm{~s})$ the ECH injection. Here, the $T_{e}$ profiles have been taken from the Thomson scattering system measurement instead of the ECE radiometer measurement since some channels of the ECE radiometer for the core region $(\mathrm{R}<1.88 \mathrm{~m})$ were not available due to a notch filter for the protection of the diagnostics from a stray power of $140 \mathrm{GHz} \mathrm{ECH}$. Note that the $T_{i}$ profiles have been taken from the discharge \#16485 since NBI blips were not applied in the discharge \#16481. In Fig. 5(a), the $n_{e}$ profile is little changed by $\mathrm{ECH}$, meaning that the onset of the QC mode was not influenced by the $n_{e}$ gradient. In Fig. 5(b), the $T_{e}$ is largely increased by $\mathrm{ECH}$ in a wide core region $(R \leq 205 \mathrm{~cm})$, but its gradient $\left(R / L_{T_{e}}\right)$ is not changed much in the region where the QC mode was measured $(184 \mathrm{~cm} \leq R \leq 191 \mathrm{~cm})$. In Fig. $5(\mathrm{c})$, the $T_{i}$ slightly drops by $\mathrm{ECH}$ in the whole region from the core to the edge. In Fig. 5(d), however, the $T_{e} / T_{i}$ is significantly increased by $\mathrm{ECH}$. All of these results suggest that the QC mode in the
ECH discharges is destabilized by the increased $T_{e} / T_{i}$ due to the ECH injection rather than by $n_{e}$ and $T_{e}$ gradients, both of which remain unchanged or slightly decrease.

Figures 5(e)-5(h) show the profiles of $n_{e}, T_{e}, T_{i}$, and $T_{e} / T_{i}$ for an ohmic discharge \#16124 at two distinct times: $t_{1}=2.4-2.5 \mathrm{~s}$ when a clear QC mode was observed and $t_{2}=5.9-6.0 \mathrm{~s}$ when the mode was fully suppressed. In the region where the QC mode was measured $(195 \mathrm{~cm} \leq R$ $\leq 202 \mathrm{~cm}), n_{e}$ at $t_{2}$ is clearly higher than that at $t_{1}$, whereas $T_{e}, T_{i}$. and $T_{e} / T_{i}$ are all slightly lower at $t_{2}$ than those at $t_{1}$. These results suggest that the QC mode is suppressed by the increased collisionality primarily due to the increased $n_{e}$, which is consistent with previous studies.

We investigate systematically the dependence of the QC mode on three local parameters: the collisionality, $T_{e} / T_{i}$, and toroidal rotation shear. For the collisionality, we use a dimensionless collisionality (known as the normalized collisionality $\nu^{*}$ ) defined by ${ }^{35}$

$$
\nu^{*} \equiv \nu_{e i} / \nu_{\mathrm{b} e}=0.00118 q Z_{\mathrm{eff}} R n_{e} / T_{e}^{2} \epsilon^{1.5},
$$



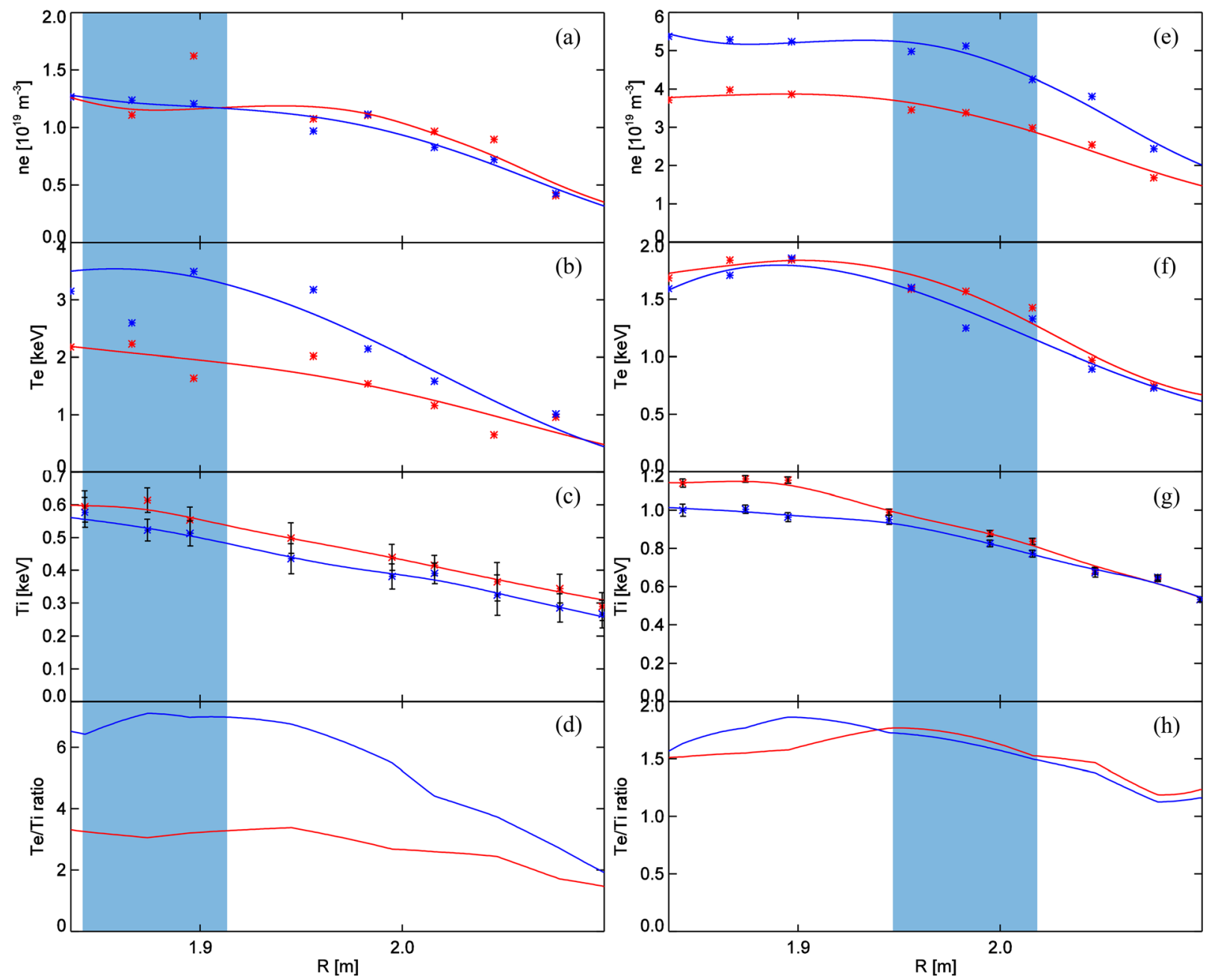

FIG. 5. Radial profiles of (a) $n_{e}$, (b) $T_{e}$, (c) $T_{i}$, and (d) $T_{e} / T_{i}$ for the ECH discharge \#16481 before (at $t=2.8 \mathrm{~s}$ colored by red) and after (at $t=3.3 \mathrm{~s}$ colored by blue) ECH injection. And profiles of (e) $n_{e}$, (f) $T_{e}$, (g) $T_{i}$, and (h) $T_{e} / T_{i}$ for the ohmic discharge \#16124 at two distinct times: low-density phase ( $n_{e}$ and $T_{e}$ at $2.4 \mathrm{~s}, T_{i}$ at $2.5 \mathrm{~s}$ colored by red) and high-density phase ( $n_{e}$ and $T_{e}$ at $5.9 \mathrm{~s}, T_{i}$ at $6.0 \mathrm{~s}$ colored by blue). Blueish regions are the area where QC modes are measured.

where $\nu_{e i}$ is the electron-ion collisionality, $\nu_{\mathrm{be}}$ is the bouncing frequency of trapped electrons in a banana orbit, $q$ is the safety factor, $Z_{\text {eff }}$ is the effective ion charge (assumed 2.0 in this analysis), $R$ is the major radius in $\mathrm{m}$, and $\epsilon$ is the inverse aspect ratio. Note that $n_{e}$ in $10^{19} \mathrm{~m}^{-3}$ and $T_{e}$ in $\mathrm{keV}$. The energy of a QC mode in the power spectrum is defined by the relative amplitude $[\mathrm{dB}]$ divided by the $1 / \mathrm{e}$ width $[\mathrm{kHz}]$ of the mode ${ }^{17}$ The dependence of the QC mode energy on $\nu^{*}$ is shown in Fig. 6(a). Here, the QC mode energy and $\nu^{*}$ have been calculated at several times for $100 \mathrm{~ms}$ after $350 \mathrm{~ms}$ from each NBI blip to avoid the effect of NBI blip. Note that for the discharge \#16481 where NBI blip was not applied, the two quantities can be calculated at more times. As $\nu^{*}$ increases, the energy of QC mode decreases in both the ECH and ohmic discharges. Figures 6(b) and 6(c) show the dependence of the QC mode energy on $T_{e} / T_{i}$ and normalized toroidal rotation shear $\left(u^{\prime}\right)$ defined by $u^{\prime}=-\left(R^{2} / V_{\mathrm{th}, i}\right) d \omega / d r$, where $V_{\mathrm{th}, i}$ is the ion thermal speed and $\omega=V_{t} / R$ is the angular velocity. ${ }^{36}$ Note that the three quantities have been calculated only at the NBI blip times since $T_{i}$ and $u^{\prime}$ were obtained by CES, which requires the NBI blip. In Fig. 6(b), the QC mode energy in the ECH discharges increases in the range of $1.6<T_{e} / T_{i}<1.9$. On the other hand, the dependence on $T_{e} / T_{i}$ in the ohmic discharges is not clear in the narrow range of $0.8 \leq T_{e} / T_{i} \leq 1.1$. For the ohmic discharges, it was difficult to make a sufficiently wide range for $T_{e} / T_{i}$. In Fig. 6(c), the QC modes are suppressed by increased $u^{\prime}$ and the critical (absolute) shear is $\sim 0.9$ for the ECH discharges and $\sim 0.3$ for the ohmic discharges. Here, it has to be noted that $\nu^{*}$ is strongly correlated to $u^{\prime}$ and it is difficult to separate the two effects on the QC mode evolution in our analysis. The higher critical $\nu^{*}$ and $u^{\prime}$ for the QC mode suppression in the ECH discharges seem to be due to the significantly higher $T_{e} / T_{i}$.

\section{Comparison with linear gyro-kinetic simulation result}

The QC mode evolution due to increased density is compared with the linear GYRO simulation result. ${ }^{30}$ The plasma parameters used for the simulation inputs for two discharges (ECH discharge \#16485 and ohmic discharge \#16124) are listed in Table I. Figure 7 shows the real frequency $\left(\omega_{r}\right)$ and the linear growth rate $(\gamma)$ of unstable mode as a function of $k_{y} \rho_{s}$ for two distinct phases at which the QC mode was 

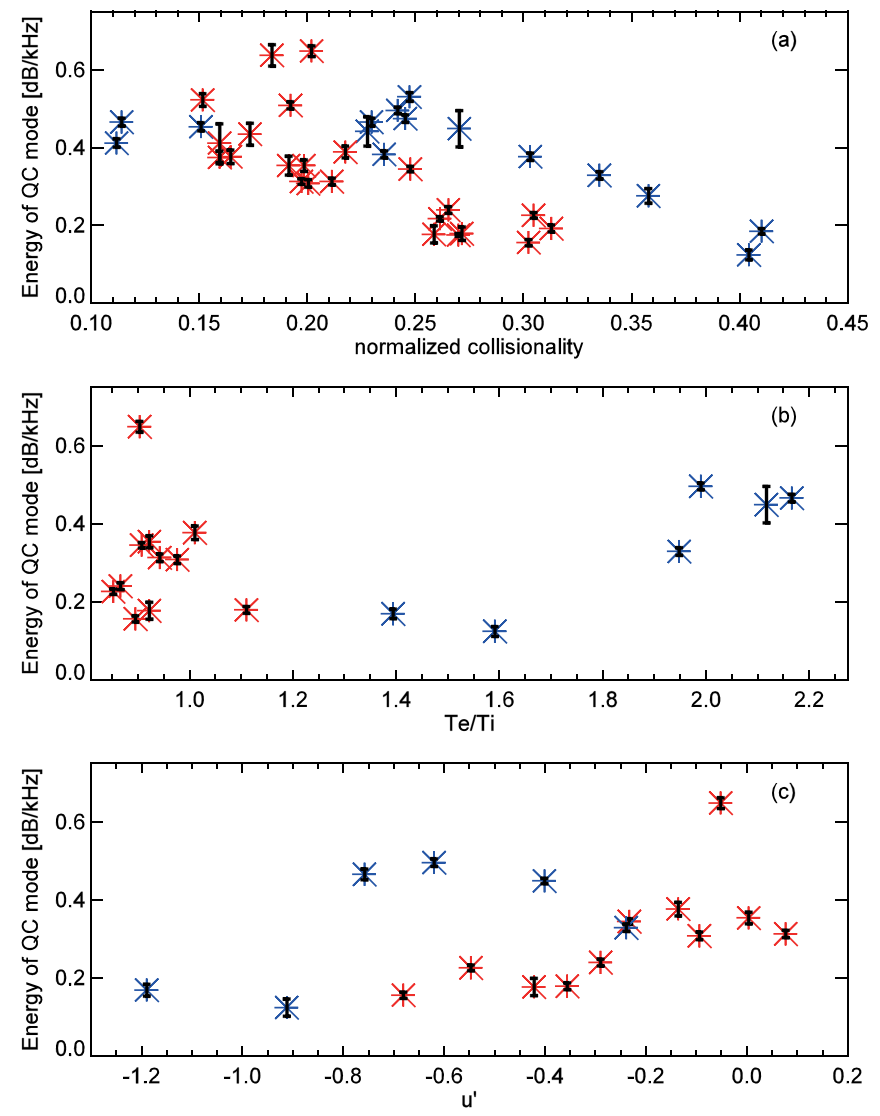

FIG. 6. The energy of QC mode versus (a) $\nu^{*}$, (b) $T_{e} / T_{i}$, and (c) $u^{\prime}$. Blue symbols indicate results for the ECH discharges \#16481 and \#16485 at $0.18 \leq r / a \leq 0.33$, and red symbols indicate results for the ohmic discharges $\# 16124$ to \#16126 at $0.44 \leq r / a \leq 0.58$.

observed and not observed, respectively, where $k_{y}$ is the poloidal wavenumber and $\rho_{s}=\sqrt{T_{e} m_{i}} /\left(Z_{i} e B\right)$ is the ion gyro-radius at electron temperature with $m_{i}$ the ion mass, $Z_{i}$ the charge number, $e$ the electron charge, and $B$ the total magnetic field strength. Note that $\omega_{r}$ and $\gamma$ are normalized to $c_{s} / a$, where $c_{s}=\sqrt{T_{e} / m_{i}}$ is the sound speed and $a$ is the minor radius. In the ECH discharge $\# 16485$, the $\omega_{r}$ at the wavenumber corresponding to the maximum $\gamma\left(k_{y} \rho_{s}\right.$ $\sim 0.6-0.7$ ) changes from the electron diamagnetic direction (indicated by positive sign) at $t=5.2 \mathrm{~s}$ to the ion direction (negative sign) at $t=6.7 \mathrm{~s}$ with an increase of $\nu^{*}$ from 0.2 to 0.9 . This result indicates that the dominant mode is TEM at low collisionality phase $(t=5.2 \mathrm{~s})$ and is ITG at high collisionality phase $(t=6.7 \mathrm{~s})$, which are consistent with results from other tokamaks. In the ohmic discharge \#16124, the $\omega_{r}$ at the wavenumber corresponding to the maximum $\gamma$ $\left(k_{y} \rho_{s}>0.9\right)$ remains in the electron direction even at the high collisionality phase $(t=5.9 \mathrm{~s})$ where the QC mode was entirely suppressed. Our further analysis indicates that the dominant mode cannot be ITG at the high collisionality phase since $a / L_{T_{i}}$ is not sufficiently large. Figure 8 shows the real frequency of the unstable mode of $k_{y} \rho_{s}=1.0$ as a function of $a / L_{T_{i}}$ and $a / L_{n_{e}}$ for the ohmic discharge \#16124 at $t=5.9 \mathrm{~s}$. It is found that the TEM to ITG transition can occur when $a / L_{T_{i}}>2.9$, which cannot be achieved in ohmic discharges without external ion heating. These results suggest that the QC mode is a TEM turbulence surviving only in low collisionality, known as the collisionless TEM (CTEM).

\section{QC MODE EVOLUTION VERSUS INTRINSIC TOROIDAL ROTATION REVERSAL}

In the ECH and ohmic L-mode plasmas, it is observed that the core toroidal rotation is gradually reversed from the co-current direction to the counter-current direction as the density increases as shown in Fig. 9. One of the theoretical models to explain the change of the toroidal intrinsic rotation is that it is mainly driven by the change of residual stress. The residual stress plays an important role to reverse the toroidal rotation direction since the change of the sign of the residual stress causes the change of the rotation direction. ${ }^{23}$ The sign of the residual stress can flip by transition between TEM and ITG since the sign of the residual stress can change when the underlying turbulence propagation direction changes. From this model, it is expected that the direction of the core intrinsic rotation is co-current direction in the TEM dominant plasmas and counter-current direction in the ITG domain plasmas.

In Fig. 9(a), the direction of the core intrinsic rotation for the ECH discharge \#16485 is reversed from the cocurrent direction to the counter-current direction with the increase of line-averaged electron density. The reversal of the core rotation direction can be explained by the TEM to ITG transition model since the QC mode is observed when the core rotation direction is the co-current direction at $\mathrm{t}=3.275 \mathrm{~s}$ in Figs. 3 and 9(a), but the QC mode disappears when it is the counter-current direction at $t=6.275 \mathrm{~s}$. However, the core rotation direction is the counter-current direction at $t=5.275 \mathrm{~s}$ where the QC mode is observed as shown in Fig. 3, which is not explained by the TEM-ITG transition model since TEM is the dominant microturbulence as shown in Figs. 7(a) and 7(b). For the discharge $\# 16124$, the core rotation direction is the counter-current direction at $t=2.5 \mathrm{~s}$ in the region where the QC mode was

TABLE I. Input parameters for linear stability analysis with GYRO for the ECH discharge \#16485 (at $5.2 \mathrm{~s}$ with QC mode and $6.7 \mathrm{~s}$ with suppressed QC mode) and ohmic discharge 16124 (at $2.4 \mathrm{~s}$ with QC mode and $5.9 \mathrm{~s}$ with suppressed QC mode). Note that $T_{i}$ and $a / L_{T_{i}}$ for the discharge \#16485 are taken at $5.3 \mathrm{~s}$ and $6.8 \mathrm{~s}$ since NBI blip was applied at $t=5.3 \mathrm{~s}$ and $6.8 \mathrm{~s}$. Also, $T_{i}$ and $a / L_{T_{i}}$ for the discharge \#16124 are taken at $2.5 \mathrm{~s}$ and $6.0 \mathrm{~s}$ since NBI blip was applied at $t=2.5 \mathrm{~s}$ and $6.0 \mathrm{~s}$

\begin{tabular}{|c|c|c|c|c|c|c|c|c|c|c|c|}
\hline Shot & $R(\mathrm{~m})$ & time (s) & $\mathrm{T}_{\mathrm{i}}(\mathrm{keV})$ & $\mathrm{T}_{\mathrm{e}}(\mathrm{keV})$ & $n_{e}\left(10^{19} / \mathrm{m}^{-3}\right)$ & $q$ & s & $a / L_{T_{i}}$ & $a / L_{T_{e}}$ & $a / L_{n_{e}}$ & $\nu_{\mathrm{ei}}$ \\
\hline 16485 & 1.875 & 5.2 & 0.60 & 2.36 & 2.17 & 1.91 & 0.10 & 0.76 & 1.97 & 0.98 & 0.05 \\
\hline 16485 & 1.875 & 6.7 & 0.56 & 1.20 & 3.42 & 1.83 & 0.10 & 1.51 & 1.29 & 0.76 & 0.29 \\
\hline 16124 & 2.00 & 2.4 & 0.87 & 1.21 & 3.21 & 1.22 & 0.72 & 0.94 & 4.64 & 1.96 & 0.26 \\
\hline 16124 & 2.00 & 5.9 & 0.81 & 1.07 & 4.74 & 1.20 & 0.58 & 1.38 & 5.16 & 3.34 & 0.48 \\
\hline
\end{tabular}



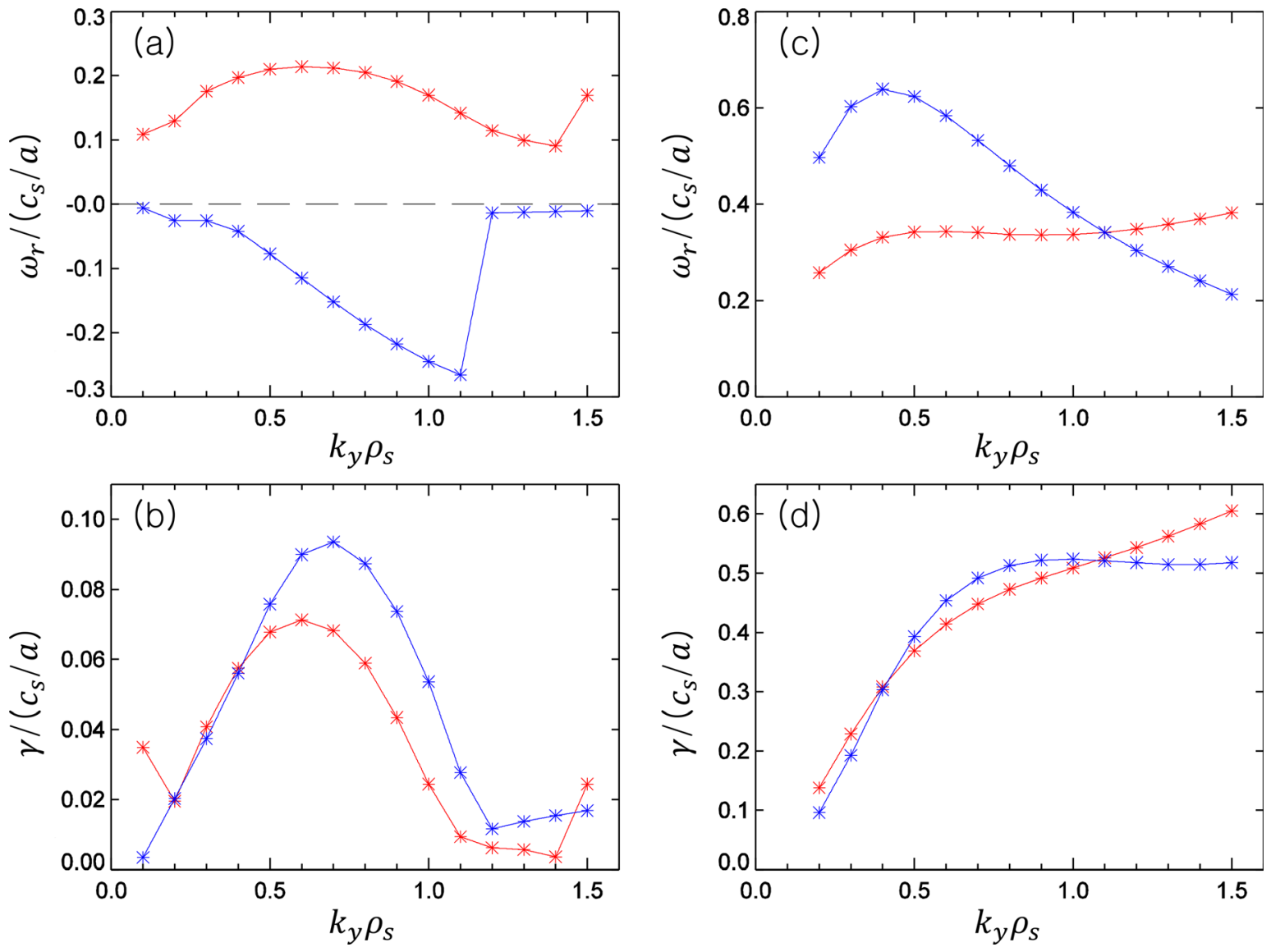

FIG. 7. GYRO simulation results with the parameters in Table I: (a) real frequency and (b) linear growth rate for the discharge \#16485 at $5.2 \mathrm{~s}$ (red) and $6.7 \mathrm{~s}$ (blue); and (c) real frequency and (d) linear growth rate for the discharge \#16124 at $2.4 \mathrm{~s}$ (red) and $5.9 \mathrm{~s}$ (blue). Note that the real frequencies and linear growth rates are normalized to $c_{s} / a$, and positive (negative) sign in the real frequency corresponds to the electron (ion) diamagnetic direction.

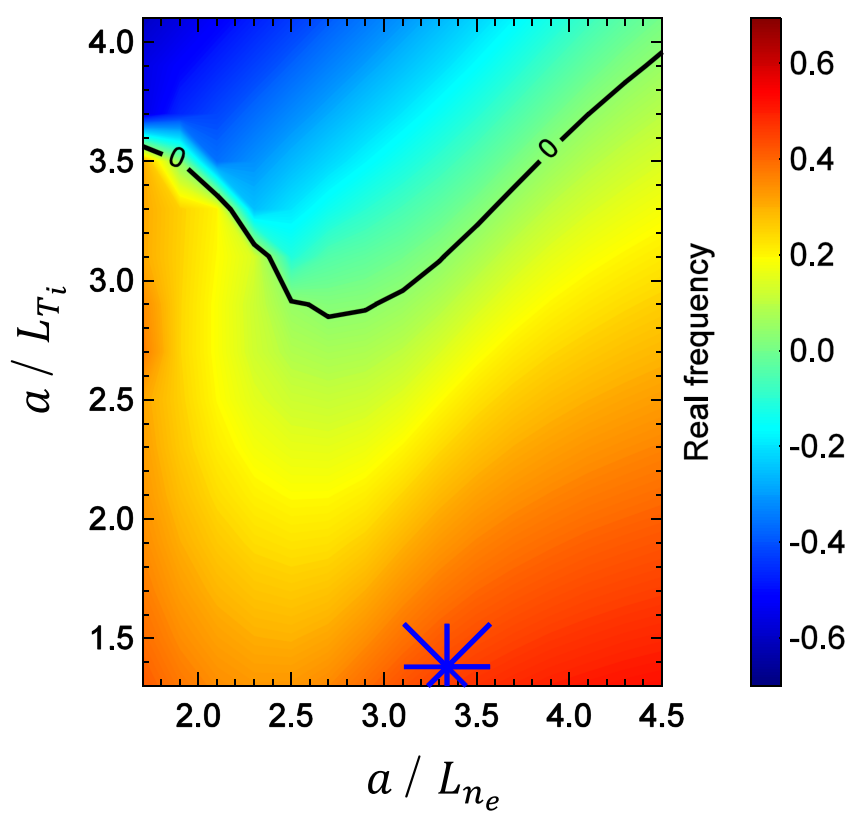

FIG. 8. The real frequency contour of the unstable mode of $k_{y} \rho_{s}=1.0$ from the GYRO simulations as a function of the density gradient and ion temperature gradient for the discharge \#16124 at $t=5.9 \mathrm{~s}$. Here, the blue color (negative) indicates ion diamagnetic direction and the red color (positive) indicates electron diamagnetic direction. The blue star represents the values of $a / L_{n_{e}}$ and $a / L_{T_{i}}$ where the experiment was conducted. measured as shown in Fig. 9(b) and TEM is the dominant micro-turbulence as shown in Figs. 7(c) and 7(d). As the time goes on, the core rotation is accelerated to the counter-current direction even the line-averaged electron density decreases at $t=4.0 \mathrm{~s}$ compared to that at $t=2.5 \mathrm{~s}$. This is not explained by the TEM-ITG transition model. Thus, it is necessary to consider other mechanisms to explain the acceleration of the core

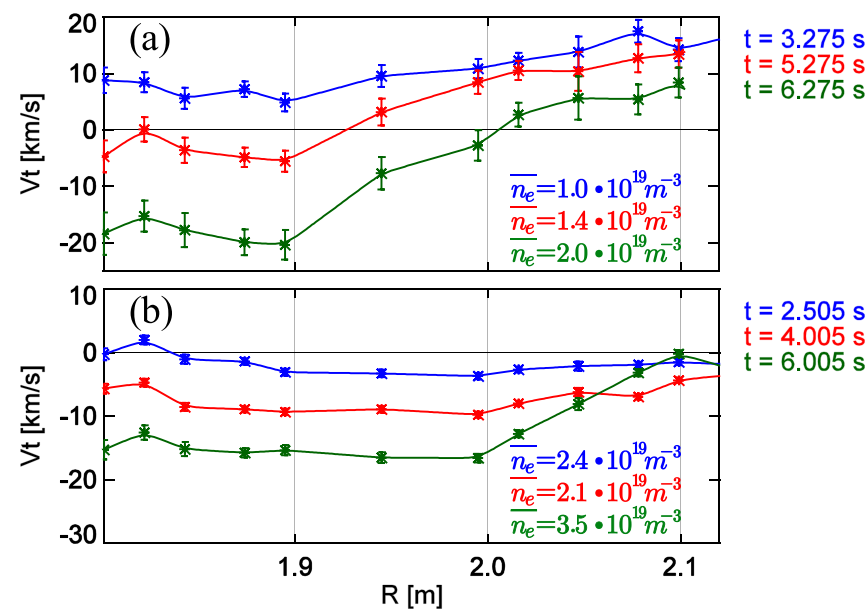

FIG. 9. Profiles of the toroidal rotation for the discharge \#16485 (a) and \#16124 (b) taken from three time slices. QC modes were observed at first two time slices and suppressed at last time slice. The positive (negative) velocity corresponds to the co-current (counter-current) rotation. 
toroidal rotation. One candidate for the driving mechanism of the core intrinsic rotation is the electron density gradient. The ASDEX upgrade experiment results ${ }^{36,37}$ showed that the core rotation is reversed from co- to counter-current direction with the increase of the density gradient. However, in a very high line-averaged electron density, the core rotation changed from counter- to co-current direction with the decrease of the density gradient. This result indicates that $R / L_{n_{e}}$ is strongly correlated to the core rotation direction in a wide range of the line-averaged electron density. In the next KSTAR campaign, we will try to control the density profile in a very high collisionality regime and to try to change the core rotation direction to the co-current direction. Also, it is known that the change of $q$ profile, ${ }^{38}$ edge region effects from scrape-off layer (SOL) flow, ${ }^{39}$ and neoclassical toroidal viscosity ${ }^{40}$ can affect the core intrinsic rotation, which will be investigated too.

\section{SUMMARY}

Electron density fluctuations measured using MIR have revealed that QC modes are destabilized in low-density phases and stabilized by increased densities in ECH and ohmic L-mode discharges in KSTAR. In the ECH discharges, QC modes are excited by ECH injection causing a significant increase of $T_{e} / T_{i}$ and reduced by increased line averaged electron density. The increased density is accompanied by the increased collisionality, reduced $T_{e} / T_{i}$, and increased toroidal rotation shear in both the ECH and ohmic discharges. The energy of the QC modes decreases gradually as the normalized collisionality increases. Although the evolutions of $T_{e} / T_{i}$ and toroidal rotation shear are strongly associated with that of the normalized collisionality, the energy of the QC modes seems to be suppressed when the $T_{e} / T_{i}$ decreases below the critical level in the ECH discharges but not to be related to $T_{e} / T_{i}$ within a narrow $T_{e} / T_{i}$ range in ohmic discharges. And the energy of the QC modes seems to be suppressed when the toroidal rotation shear increases above a critical level. The critical level of the toroidal rotation shear for the QC mode suppression is higher in the ECH discharges than in the ohmic discharges probably due to higher $T_{e} / T_{i}$.

Gyro-kinetic simulations have shown that the dominant modes are TEM in low-density phases where the QC modes were observed for both the ECH and ohmic discharges. In high-density phases where the QC modes were suppressed, the dominant mode is ITG for the ECH discharges, whereas TEM for the ohmic discharges. In ohmic heated plasmas, the dominant micro-turbulence cannot be ITG at the high collisionality phase since the ion temperature gradient is not sufficiently high. These results together with the measurements suggest that the QC mode is close to collisionless TEM turbulence.

It has been observed that the core toroidal rotation is accelerated to counter-current direction when the QC modes are suppressed with the increase of collisionality. A theoretical model predicted that the direction of the core toroidal intrinsic rotation is changed from co-current to countercurrent direction by the TEM to ITG transition when the plasma collisionality increases in L-mode plasmas without external torque. However, the TEM-ITG transition cannot fully explain the change of the core intrinsic rotation direction observed in our measurements. The core intrinsic rotation direction is the counter-current direction in the plasmas where the QC modes are destabilized, but ITG cannot be destabilized. Also, the core intrinsic rotation is accelerated to the counter-current direction even if the line-averaged electron density decreases. Hence, it is necessary to consider other mechanisms to explain the evolutions of the core toroidal intrinsic rotation such as the electron density gradient.

\section{ACKNOWLEDGMENTS}

This work was supported by the National Research Foundation of Korea under Contract Nos. NRF2017M1A7A1A03064231 and NRF-2014M1A7A1A03029865, and the Ministry of Science, ICT, and Future Planning of Korea under the KSTAR project and Contract No. NFRI-EN1741-3.

${ }^{1}$ W. Horton, Rev. Mod. Phys. 71, 735 (1999).

${ }^{2}$ M. Kikuchi and M. Azumi, Rev. Mod. Phys. 84, 1807 (2012).

${ }^{3}$ F. Romanelli, Phys. Fluids B 1, 1018 (1989).

${ }^{4}$ P. Mantica, D. Strintzi, T. Tala, C. Giroud, T. Johnson, H. Leggate, E. Lerche, T. Loarer, A. G. Peeters, A. Salmi et al., Phys. Rev. Lett. 102 , 175002 (2009)

${ }^{5}$ P. Mantica, C. Angioni, C. Challis, G. Colyer, L. Frassinetti, N. Hawkes, T. Johnson, M. Tsalas, P. C. deVries, J. Weiland et al., Phys. Rev. Lett. 107, 135004 (2011).

${ }^{6}$ T. Dannert and F. Jenko, Phys. Plasmas 12, 072309 (2005)

${ }^{7}$ X. Garbet, P. Mantica, C. Angioni, E. Asp, Y. Baranov, C. Bourdelle, R. Budny, F. Crisanti, G. Cordey, L. Garzotti et al., Plasma Phys. Controlled Fusion 46, B557 (2004).

${ }^{8}$ G. T. Hoang, C. Bourdelle, X. Garbet, G. Giruzzi, T. Aniel, M. Ottaviani, W. Horton, P. Zhu, and R. V. Budny, Phys. Rev. Lett. 87, 125001 (2001).

${ }^{9}$ F. Jenko and W. Dorland, Phys. Rev. Lett. 89, 225001 (2002).

${ }^{10}$ F. Jenko, D. Told, T. Görler, J. Citrin, A. Bañón Navarro, C. Bourdelle, S. Brunner, G. Conway, T. Dannert, and H. Doerk, Nucl. Fusion 53, 073003 (2013).

${ }^{11}$ J. E. Rice, A. Ince-Cushman, J. S. deGrassie, L.-G. Eriksson, Y. Sakamoto, A. Scarabosio, A. Bortolon, K. H. Burrell, B. P. Duval, C. Fenzi-Bonizec et al., Nucl. Fusion 47, 1618 (2007).

${ }^{12}$ J. E. Rice, B. P. Duval, M. L. Reinke, Y. A. Podpaly, A. Bortolon, R. M. Churchill, I. Cziegler, P. H. Diamond, A. Dominguez, P. C. Ennever et al., Nucl. Fusion 51, 083005 (2011).

${ }^{13}$ T. Fülöp and H. Nordman, Phys. Plasmas 16, 032306 (2009).

${ }^{14}$ X. Garbet, L. Garzotti, P. Mantica, H. Nordman, M. Valovic, H. Weisen, and C. Angioni, Phys. Rev. Lett. 91, 035001 (2003).

${ }^{15}$ V. A. Vershkov, V. F. Andreev, A. A. Borschegovskiy, V. V. Chistyakov, M. M. Dremin, L. G. Eliseev, E. P. Gorbunov, S. A. Grashin, A. V. Khmara, A. Y. Kislov et al., Nucl. Fusion 51, 094019 (2011).

${ }^{16} \mathrm{H}$. Arnichand, R. Sabot, S. Hacquin, A. Krämer-Flecken, X. Garbet, J. Citrin, C. Bourdelle, G. Hornung, J. Bernardo, C. Bottereau et al., Nucl. Fusion 54, 123017 (2014).

${ }^{17}$ H. Arnichand, R. Sabot, S. Hacquin, A. Krämer-Flecken, C. Bourdelle, J. Citrin, X. Garbet, J. C. Giacalone, R. Guirlet, J. C. Hillesheim et al., Nucl. Fusion 55, 093021 (2015).

${ }^{18}$ H. Arnichand, J. Citrin, S. Hacquin, R. Sabot, A. Krämer-Flecken, X. Garbet, C. Bourdelle, C. Bottereau, F. Clairet, J. C. Giacalone et al., Plasma Phys. Controlled Fusion 58, 014037 (2016).

${ }^{19}$ W. L. Zhong, Z. B. Shi, Z. J. Yang, G. L. Xiao, Z. C. Yang, B. Y. Zhang, P. W. Shi, H. R. Du, X. M. Pan, R. B. Zhou et al., Phys. Plasmas 23, 060702 (2016).

${ }^{20}$ W. Lee, J. Leem, G. S. Yun, H. K. Park, J. A. Lee, Y. B. Nam, Y. U. Nam, W. H. Ko, J. H. Jeong, and Y. S. Bae, J. Instrum. 8, C10018 (2013).

${ }^{21}$ W. Lee, J. Leem, J. A. Lee, Y. B. Nam, M. Kim, G. S. Yun, H. K. Park, Y. G. Kim, H. Park, K. W. Kim et al., Nucl. Fusion 54, 023012 (2014).

${ }^{22}$ W. Lee, J. Leem, G. S. Yun, H. K. Park, S. H. Ko, W. X. Wang, R. V. Budny, N. C. Luhmann, Jr., and K. W. Kim, Rev. Sci. Instrum. 87, 11E134 (2016). 
${ }^{23}$ P. H. Diamond, C. J. McDevitt, Ö. D. Gürcan, T. S. Hahm, W. X. Wang, E. S. Yoon, I. Holod, Z. Lin, V. Naulin, and R. Singh, Nucl. Fusion 49, 045002 (2009).

${ }^{24}$ P. H. Diamond, Y. Kosuga, Ö. D. Gürcan, C. J. McDevitt, T. S. Hahm, N. Fedorczak, J. E. Rice, W. X. Wang, S. Ku, J. M. Kwon et al., Nucl. Fusion 53, 104019 (2013).

${ }^{25}$ S. H. Jeong, K. D. Lee, Y. Kogi, K. Kawahata, Y. Nagayama, A. Mase, and M. Kwon, Rev. Sci. Instrum. 81, 10D922 (2010).

${ }^{26}$ J. H. Lee, S. T. Oh, and H. M. Wi, Rev. Sci. Instrum. 81, 10 D528 (2010).

${ }^{27}$ Y. U. Nam and K. D. Lee, Rev. Sci. Instrum. 79, 10 E705 (2008).

${ }^{28}$ J.-W. Juhn, K. C. Lee, H. M. Wi, Y. S. Kim, and Y. U. Nam, Rev. Sci. Instrum. 87, 11E131 (2016).

${ }^{29}$ K. Won-Ha, O. Seungtae, and K. Myeun, IEEE Trans. Plasma Sci. 38, 996 (2010).

${ }^{30}$ J. Candy and R. E. Waltz, J. Comput. Phys. 186, 545 (2003).

${ }^{31}$ F. Ryter, C. Angioni, A. G. Peeters, F. Leuterer, H.-U. Fahrbach, and W. Suttrop (ASDEX Upgrade Team), Phys. Rev. Lett. 95, 085001 (2005).

${ }^{32}$ A. G. Peeters, C. Angioni, M. Apostoliceanu, F. Jenko, F. Ryter, and the ASDEX Upgrade Team, Phys. Plasmas 12, 022505 (2005).
${ }^{33}$ C. Angioni, A. G. Peeters, F. Jenko, and T. Dannert, Phys. Plasmas 12, 112310 (2005).

${ }^{34}$ J.-M. Kwon, L. Qi, S. Yi, and T. S. Hahm, Comput. Phys. Commun. 215, 81 (2017).

${ }^{35}$ G. D. Conway, C. Angioni, R. Dux, F. Ryter, A. G. Peeters, J. Schirmer, C. Troester, CFN Reflectometry Group, and the ASDEX Upgrade Team, Nucl. Fusion 46, S799 (2006).

${ }^{36}$ R. M. McDermott, C. Angioni, G. D. Conway, R. Dux, E. Fable, R. Fischer, T. Pütterich, F. Ryter, E. Viezzer, and the ASDEX Upgrade Team, Nucl. Fusion 54, 043009 (2014).

${ }^{37}$ C. Angioni, R. M. McDermott, F. J. Casson, E. Fable, A. Bottino, R. Dux, R. Fischer, Y. Podoba, T. Pütterich, F. Ryter et al., Phys. Rev. Lett. 107, 215003 (2011)

${ }^{38}$ W. X. Wang, T. S. Hahm, S. Ethier, L. E. Zakharov, and P. H. Diamond, Phys. Rev. Lett. 106, 085001 (2011).

${ }^{39}$ B. LaBombard, J. E. Rice, A. E. Hubbard, J. W. Hughes, M. Greenwald, J. Irby, Y. Lin, B. Lipschultz, E. S. Marmar, C. S. Pitche et al., Nucl. Fusion 44, 1047 (2004).

${ }^{40}$ Y. Sun, K. C. Shaing, Y. Liang, T. Casper, A. Loarte, B. Shen, and B. Wan, Nucl. Fusion 53, 093010 (2013). 